\title{
Elevated systemic levels of the matrix metalloproteinase inhibitor TIMP-1 correlate with clinical markers of cachexia in patients with chronic pancreatitis and pancreatic cancer
}

\author{
Olga Prokopchuk ${ }^{1 * \dagger}$ (D), Barbara Grünwald ${ }^{2 \dagger}$, Ulrich Nitsche ${ }^{1}$, Carsten Jäger ${ }^{1}$, Oleksii L. Prokopchuk ${ }^{3}$,
} Elaine C. Schubert ${ }^{4}$, Helmut Friess ${ }^{1}$, Marc E. Martignoni ${ }^{1}$ and Achim Krüger ${ }^{2}$

\begin{abstract}
Background: Tissue inhibitor of metalloproteinases-1 (TIMP-1) is a candidate diagnostic and prognostic biomarker for pancreatic ductal adenocarcinoma (PDAC). Here, we determined the possible association of systemic TIMP-1 levels with cachexia and jaundice, two common PDAC-associated conditions.

Methods: Plasma TIMP-1 was measured by ELISA in patients diagnosed with PDAC $(n=36)$ and chronic pancreatitis (CP) $(n=25)$. Patients without pancreatic pathologies and known malignancies of other origin served as controls $(n=13)$. TIMP-1 levels in these patients were tested for asscociation with jaundice and chachexia, and furthermore correlated with cachexia-related clinical parameters such as weight loss and ferritin, parameters of lung function, hemoglobin and liver synthesis parameters.

Results: TIMP-1 plasma levels were mostly higher in CP and PDAC patients with concomitant jaundice or cachexia. Elevated plasma TIMP-1 levels were also associated with clinical cachexia markers, including absolute and relative values of weight loss and lung function, as well as ferritin, hemoglobin, and cholinesterase levels. TIMP-1 levels significantly correlated with cachexia only in patients without jaundice. Jaundice also impaired the use of TIMP-1 as a prognostic marker in cancer patients. Relating to cachexia status alone, a slightly improved association of TIMP-1 levels with survival of PDAC patients was observed.
\end{abstract}

Conclusion: This retrospective study reports for the first time that plasma levels of TIMP-1 are associated with pancreatic lesion-induced cachexia in patients without jaundice. TIMP-1 is counterindicated as a survival marker in patients with jaundice.

Keywords: TIMP-1, Cachexia biomarker, Pancreas, Jaundice

\footnotetext{
* Correspondence: olga.prokopchuk@tum.de

${ }^{\dagger}$ Equal contributors

${ }^{1}$ Klinik und Poliklinik für Chirurgie, Klinikum rechts der Isar, Technische

Universität München, Ismaninger Str. 22, 81675 Munich, Germany

Full list of author information is available at the end of the article
} 


\section{Background}

Tissue inhibitor of metalloproteinases-1 (TIMP-1) correlates with tumor progression [1-5], and elevated levels of TIMP-1 in tumor tissue and in peripheral blood are associated with poor clinical outcome in numerous malignancies, including colorectal cancer [1, 2, 6, 7], breast cancer $[3,5]$, gastric cancer $[8,9]$, non-small cell lung cancer [10], and esophageal cancer [4]. TIMP-1 is increasingly recognized as a molecule with a variety of pro-tumorigenic functions, e.g. TIMP-1 can bind to the tetraspanin CD63 and promote liver metastases via both, host-mediated mechanisms as well as direct effects on tumor cell aggressiveness $[1,11-13]$. In specific, TIMP1 signaling via CD63 leads to activation of hepatic stellate cells, which create a pre-metastatic niche in the liver allowing efficient metastasis to this organ [14], and also induces a tumor-promoting stress response in tumor cells [11].

Several independent studies have reported TIMP-1 to be of prognostic and diagnostic value for pancreatic ductal adenocarcinoma (PDAC) $[15,16]$. TIMP-1 transcripts are found in both stroma and tumor cells of human PDAC [17]. TIMP-1 expression positively correlates with the degree of desmoplasia in the tumor stroma [17], as well as with de-differentiation of pancreatic tumor cells [18]. In experimental mouse models of chronic pancreatitis $(\mathrm{CP})$, TIMP-1 mRNA expression in the pancreas increases with disease progression, suggesting an important role of TIMP-1 in pancreatic fibrosis [19]. Expression of TIMP-1 is not only increased in early pancreatic lesions such as $\mathrm{CP}$ but also in pancreatic intra-epithelial neoplasia and late PDAC [14]. Importantly, plasma levels of TIMP-1 protein were found to be significantly elevated in PDAC and $\mathrm{CP}$ patients in an unbiased system-wide proteomics approach [20, 21], which further supports its potential usefulness as a diagnostic tumor marker.

PDAC is often associated with jaundice, and it was shown that TIMP-1 levels are significantly elevated in patients with PDAC-associated jaundice as well as with jaundice due to non-malignant conditions such as gallstones [22]. Cachexia is another common condition associated with progression of pancreatic lesions, occurring in almost $40 \%$ of patients with CP and PDAC, and is a strong prognostic factor for PDAC patients [23]. Association of TIMP-1 with cachexia was observed in several established animal cancer cachexia models including the rat hepatoma cancer cachexia model [24], Wistar rats bearing subcutaneous Walker256 carcinomas [25], and CD2F1 mice bearing C26 adenocarcinomas [26]. These data suggest that PDAC-associated conditions may influence systemic TIMP-1 levels, potentially through fibrotic remodeling and inflammation. As such, these conditions may either benefit or hamper the clinical usefulness of TIMP-1 as a biomarker. So far, there is no consensus whether PDAC patients with concomitant jaundice have to be excluded from use of TIMP-1 as a prognostic marker for pancreatic cancer patients. Further, the impact of cachexia on the usefulness of TIMP-1 as clinical progression and survival marker has not yet been evaluated. It is also not clear whether TIMP-1 alone or in combination with cachexia and jaundice may have improved prognostic value in PDAC.

In this retrospective study, we explore the impact of cachexia and jaundice on the diagnostic and prognostic value of TIMP-1, in CP and PDAC. We further determine the usefulness of plasma TIMP-1 levels as a cachexia biomarker. We report that TIMP-1 was associated with clinical markers of cachexia and with presence of cachexia in our cohort. While TIMP-1 was counterindicated as a marker in combination with jaundice, combining TIMP-1 with cachexia represents a promising combination of prognostic parameters.

\section{Methods}

\section{Patients and tissue biopsies}

This study was approved by the Ethics Committee of the Medical Faculty of the Technical University of Munich (Germany; \#1946/07), and written consent was obtained from all participants before surgery or before blood sampling.

\section{Clinical parameters assessment}

The analysis was conducted on a pseudonymized data set. The study population comprised patients suspicious for pancreatic cancer or chronic pancreatitis between 2008 and 2015 in the Department of Surgery, Klinikum Rechts der Isar, Munich, who agreed to participate in the study. Plasma samples were taken after written informed consent. The diagnosis was verified by postoperative definitive histological examination, or, in patients without surgery, by cytology or clinical/radiological information, to the best of our knowledge. Weight was measured at the time of admission to the hospital. Height and weight histories over the six months preceeding admission were collected by OP and OLP. Jaundice was defined as a serum total bilirubin level $\geq 2 \mathrm{mg} /$ dl. Cachexia was defined as loss of more than $10 \%$ of the original body weight within the last six months before scheduled surgery. We modified the International Classification Framework definition of cachexia proposed by Fearon and co-workers [27] and extended the cut-off point for cachexia to $10 \%$ as described previously [23, 28-30], to unambiguously distinguish between patients with and without cachexia. This criterion was demonstrated to influence survival and performance status in PDAC patients [31, 32]. Patients without tumor diagnosis and history of chronic pancreatitis served as controls. The control samples were obtained from patients attending the same hospital. Spirometry provided a measurement of 
the lung function, as determined by forced vital capacity (FVC) and the forced expiratory volume in $1 \mathrm{~s}\left(\mathrm{FEV}_{1}\right)$, and was conducted as previously described [32].

\section{Laboratory examinations}

Blood samples were analyzed at the Institute of Clinical Chemistry and Pathobiochemistry, Klinikum rechts der Isar, Munich, according to standard operating procedures. Blood was collected in a $9 \mathrm{ml}$ EDTA tube, one $2.9 \mathrm{ml}$ coagulation tube and one $9 \mathrm{ml}$ serum tube (S-Monovette, Sarstedt, Nümbrecht, Germany), and mixed immediately by gently inverting the tube. Hemoglobin levels were determined by the sodium lauryl sulfate hemoglobin detection method, and leucocytes counts were determined by flow cytometry with integrated hydrodynamic focusing, using Sysmex XE 5000-2 or Sysmex XT 2000i hematology analyzers (Norderstedt, Germany). A photometric diazonium-based test was used to measure serum bilirubin. A photometric biuret-based test was used for measurement of serum protein and the bromcresol green reaction test was used to determine serum albumin. Serum concentrations of CRP were measured with an immunoturbidimetric assay. Cancer antigen 19-9 (CA 19-9) was evaluated by an electrochemiluminescence immunoassay. The measurements of bilirubin, protein, albumin, CRP and CA 19-9 (e 602 module) were performed on a Cobas 8000 platform (Roche Diagnostics, Mannheim, Germany).

\section{Measurement of fat and muscle tissue on computed tomography scans}

Measurement of fat and muscle tissue on computed tomography scans was performed as described previously [33]. Patients received a contrast-enhanced computed tomography (CT) scan for initial cancer staging or validation of $\mathrm{CP}$ or routine diagnostic purposes. We quantified skeletal muscle and fat thicknesses, as well as M. psoas crosssectional area. A venous phase of a CT scan of the abdomen was chosen. Six different values were taken, as

Table 1 Clinical parameters of patients

\begin{tabular}{|c|c|c|c|c|c|c|c|}
\hline & \multirow[t]{2}{*}{ Control } & \multicolumn{3}{|l|}{ PDAC } & \multicolumn{3}{|l|}{$C P$} \\
\hline & & non-cachexia & cachexia & $p$ & non-cachexia & cachexia & $p$ \\
\hline $\mathrm{N}$ & 13 & 23 & 13 & & 19 & 6 & ns \\
\hline Female & $6(46 \%)$ & $11(48 \%)$ & $6(46 \%)$ & ns & $10(53 \%)$ & $2(33 \%)$ & \\
\hline Male & 7 (54\%) & $12(52 \%)$ & 7 (54\%) & & $9(47 \%)$ & $4(67 \%$ & \\
\hline Age, years & $63(19-78)$ & $68(41-88)$ & $61(52-77)$ & ns & $50(36-77)$ & $49(44-73)$ & ns \\
\hline \multirow[t]{4}{*}{ UICC stage } & \multirow[t]{4}{*}{$\mathrm{nr}$} & \multicolumn{2}{|l|}{ | 2 (9\%) } & \multirow[t]{4}{*}{ ns } & \multirow[t]{4}{*}{$\mathrm{nr}$} & \multirow[t]{4}{*}{$\mathrm{nr}$} & \\
\hline & & || $16(70 \%)$ & || 5 (38\%) & & & & \\
\hline & & III $2(9 \%)$ & III 1 (8\%) & & & & \\
\hline & & IV $3(12 \%)$ & IV 7 (54\%) & & & & \\
\hline ASA I & $18 \%$ & $7 \%$ & $9 \%$ & \multirow[t]{3}{*}{ ns } & \multicolumn{2}{|l|}{$23 \%$} & ns \\
\hline$\|$ & $73 \%$ & $64 \%$ & $64 \%$ & & $46 \%$ & $67 \%$ & \\
\hline III & $9 \%$ & $29 \%$ & $27 \%$ & & $31 \%$ & $33 \%$ & \\
\hline Jaundice & $0(0 \%)$ & $5(22 \%)$ & $5(38 \%)$ & ns & $0(0 \%)$ & $1(17 \%)$ & ns \\
\hline Weight loss, kg & $0.0(0)$ & $2(0-8)$ & $12,5(6-34)$ & $<0.001 \#$ & $0(0-6)$ & $12,5(8-40)$ & $<0.001 \# \#$ \\
\hline Weight loss, \% & $0.0(0)$ & $3.0(0-9.2)$ & $14.0(10.0-26.6)$ & $<0.001 \#$ & $0(0-9.1)$ & $15.7(11.1-28.6)$ & $<0.001 \# \#$ \\
\hline Perirenal fat, mm & $15.2(6.7-32.5)$ & $11.0(2.0-39.0)$ & $11.6(4.0-26.2)$ & ns & $6(2.5-22.4)$ & $7.0(5.2-19.1)$ & $0.013 \S \S$ \\
\hline M. erector spinae, $\mathrm{mm}$ & $36.2(28.9-39.8)$ & $30.3(18.7-39.1)$ & $31.1(26.0-41.4)$ & ns & $32.1(24.3-47.4)$ & $40.5(36.7-45.1)$ & 0.019\#\# \\
\hline \multirow[t]{2}{*}{ M. psoas, mm } & \multirow[t]{2}{*}{$37.0(30.6-53.8)$} & \multirow[t]{2}{*}{$38.0(25.1-43.5)$} & \multirow[t]{2}{*}{$32.8(25.0-39.5)$} & \multirow[t]{2}{*}{ ns } & \multirow[t]{2}{*}{$37.9(20.7-51.2)$} & \multirow[t]{2}{*}{$28.8(22.6-35.7)$} & $0.030^{\circ \circ}$ \\
\hline & & & & & & & 0.039\#\# \\
\hline \multirow[t]{2}{*}{ M. psoas area, mm² } & \multirow{2}{*}{$\begin{array}{l}1044,2 \\
(876,2-1997,0)\end{array}$} & \multirow{2}{*}{$\begin{array}{l}1022,1 \\
(608,7-1371,8)\end{array}$} & \multirow[t]{2}{*}{$702,1(527,3-1075,2)$} & $0.035^{\circ}$ & \multirow[t]{2}{*}{$943,5(345,7-1971,5)$} & \multirow[t]{2}{*}{$714,4(565,9-931,3)$} & \\
\hline & & & & $0.031 \#$ & & & \\
\hline $\begin{array}{l}\text { Subcutaneous fat } \\
\text { medial, mm }\end{array}$ & $17.8(6.7-62.6)$ & $14.0(3.0-60.6)$ & $20.7(1.1-50.4)$ & ns & $8.2(0.6-62.3)$ & $6.3(2.5-31.2)$ & \\
\hline $\begin{array}{l}\text { Subcutaneous fat } \\
\text { lateral, mm }\end{array}$ & $45.0(29.1-92.0)$ & $37.1(14.9-77.5)$ & $38.3(7.7-72.4)$ & ns & $33.5(4.3-94.5)$ & $34.6(22.3-45.3)$ & \\
\hline
\end{tabular}

Values are demonstrated as median (minimum-maximum)

ns not significant; $\mathrm{nr}$ not relevant

- PDAC with cachexia versus control; \# PDAC without cachexia versus PDAC with cachexia

$\S \S \mathrm{CP}$ without cachexia versus control; ${ }^{\circ} \mathrm{CP}$ with cachexia versus control; \#\# CP without cachexia versus $\mathrm{CP}$ with cachexia 


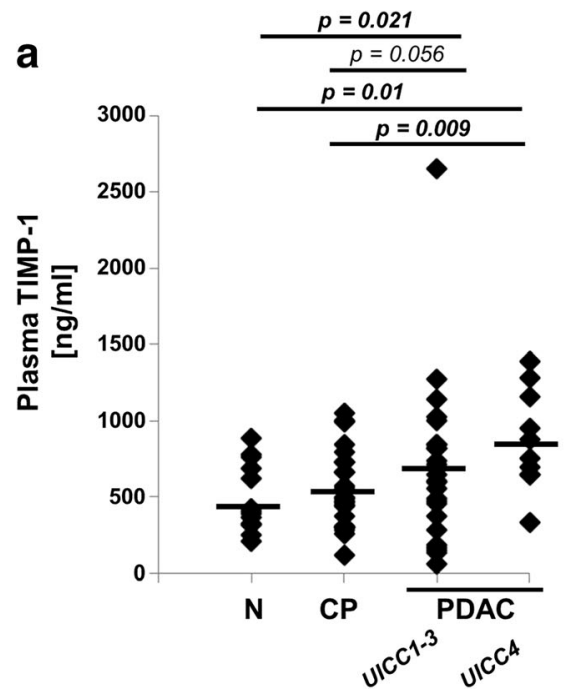

b

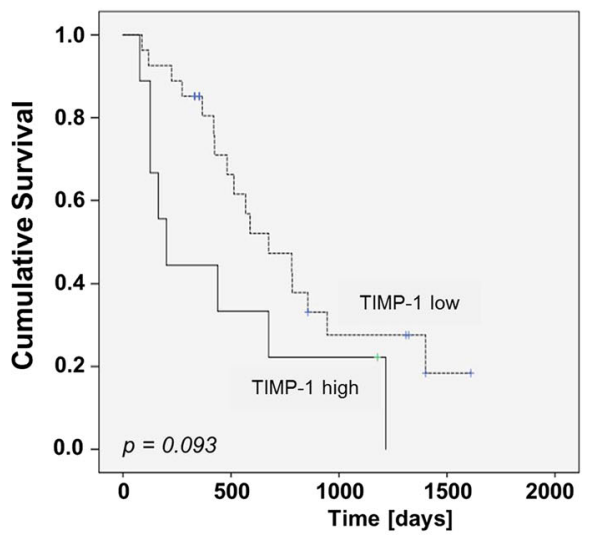

Fig. 1 a Dot plots for plasma levels of TIMP-1 protein analyzed by ELISA in healthy individuals (control, $n=13)$, CP patients ( $n=25)$, PDAC patients (UICC 1-3 $(n=26)$, and UICC $4(n=10)$ ). Shapiro-Wilks testing in combination with visual inspection of the histograms showed that samples were not normally distributed. Groups were compared using nonparametric Mann-Whitney $U$ test for independent variables. b Relation of plasma TIMP-1 to Kaplan Meier Overall Survival (OS) curves in PDAC patients. The optimal cut-off value of plasma TIMP-1 levels at $842 \mathrm{ng} / \mathrm{ml}$ was determined by maximally selected log-rank statistics. To consider multiple test issues within these analyses, the R-function maxstat.test was employed. For the cut-off at $842 \mathrm{ng} / \mathrm{ml}$ the Kaplan Meier curves were generated. Median OS for patients with low plasma TIMP-1 levels $(n=26): 453$ days [440-768] 95\% Cl. Median OS for patients with high plasma TIMP-1 levels $(n=9)$ : 202 days [117-817] 95\% Cl

previously described [32]: thickness of the perirenal fat, thickness of the medial subcutaneous fat; thickness of the lateral subcutaneous fat; thickness of muscles measured at two different locations (musculus erector spinae and musculus psoas), and musculus psoas area (measured as described previously [32]).

\section{ELISA}

Blood samples were collected, and plasma was obtained within $30 \mathrm{~min}$ by centrifugation of whole blood for $15 \mathrm{~min}$ at 1000 g. Plasma samples were immediately snap-frozen in liquid nitrogen and stored at $-80{ }^{\circ} \mathrm{C}$. TIMP-1 levels in plasma were determined using the DuoSet ELISA kit (R\&D Systems) according to the manufacturer's instructions. Each sample was analyzed in triplicate. Results of the ELISA were analyzed using ReaderFit.

\section{Statistical analyses}

Statistical analysis was performed using the statistical software SPSS version 23.0 (Chicago, IL, USA). Associations between quantitative variables were tested by Spearman correlations. Normal distribution was tested by ShapiroWilk tests and visual inspection of the histograms. Groups were compared using Student's t-test for independent samples in the case of normal distribution, or nonparametric Mann-Whitney test for independent variables in the absence of normal distribution. To derive optimal cut-off values of plasma TIMP-1 levels, maximally selected logrank statistics performed by $\mathrm{R}$ Software version 2.13.0 (R Foundation for Statistical Computing, Vienna, Austria) were used. Additionally, the R-function maxstat.test was employed [34]. Time-dependent survival probabilities were estimated with the Kaplan-Meier method, and the log-rank test was used to compare independent subgroups.

Table 2 Correlations between plasma TIMP-1 and parameters of tumor load in pancreatic cancer patients

\begin{tabular}{|c|c|c|c|c|c|c|c|c|c|c|}
\hline & & $\begin{array}{l}\top \\
(1-4)\end{array}$ & $\begin{array}{l}N \\
(0,1)\end{array}$ & $\begin{array}{l}\text { Number of } \\
\text { positive lymph } \\
\text { nodes }\end{array}$ & $\begin{array}{l}\text { Number of } \\
\text { removed lymph } \\
\text { nodes }\end{array}$ & $\begin{array}{l}\text { Liver } \\
\text { metastases } \\
\text { (yes or no) }\end{array}$ & $\begin{array}{l}\text { Distant metastases } \\
\text { (yes or no) }\end{array}$ & $\begin{array}{l}\text { UICC } \\
(1-4)\end{array}$ & $\begin{array}{l}\text { Grading } \\
(1-4)\end{array}$ & $\begin{array}{l}\text { Resection status } \\
(\mathrm{RO} 0, \mathrm{R} 1)\end{array}$ \\
\hline \multirow[t]{3}{*}{$\begin{array}{l}\text { Plasma TIMP-1, } \\
\mathrm{ng} / \mathrm{ml}\end{array}$} & $\begin{array}{l}\text { Spearman's } \\
\text { correlation }\end{array}$ & .138 & -.061 & -.189 & .200 & .375 & .160 & .222 & -.026 & .049 \\
\hline & $\begin{array}{l}\text { Significance } \\
\text { (2-tailed) }\end{array}$ & .491 & .768 & .355 & .326 & .024 & .381 & .194 & .887 & .821 \\
\hline & $\mathrm{N}$ & 27 & 26 & 26 & 26 & 36 & 32 & 36 & 32 & 24 \\
\hline
\end{tabular}




\section{Results}

\section{Patient cohort}

Plasma samples from 74 patients (PDAC, $n=36$; CP, $n=25$, healthy controls, $n=13$ ) were analyzed. PDAC and control patients were over 60 years old, while the median age of $\mathrm{CP}$ patients was 14 years less than of the control patients $(p=0.527)$, and 19 years younger than PDAC patients $(p<0.001)$. Of these, 13 PDAC patients
(36\%) and $6 \mathrm{CP}$ patients (24\%) were classified as cachectic, as defined in the methods section. Most of the PDCA patients without cachexia (70\%) presented with the International Union Against Cancer (UICC) tumor stage 2 (defined as T3, N0, M0 or T1-3, N1, M0 [34, 35]). PDAC patients with cachexia presented mostly with UICC stage 4 (54\%; defined as any T, any N, M1 [35, 36]). Of the 36 PDAC patients, 10 (28\%) presented with jaundice. There

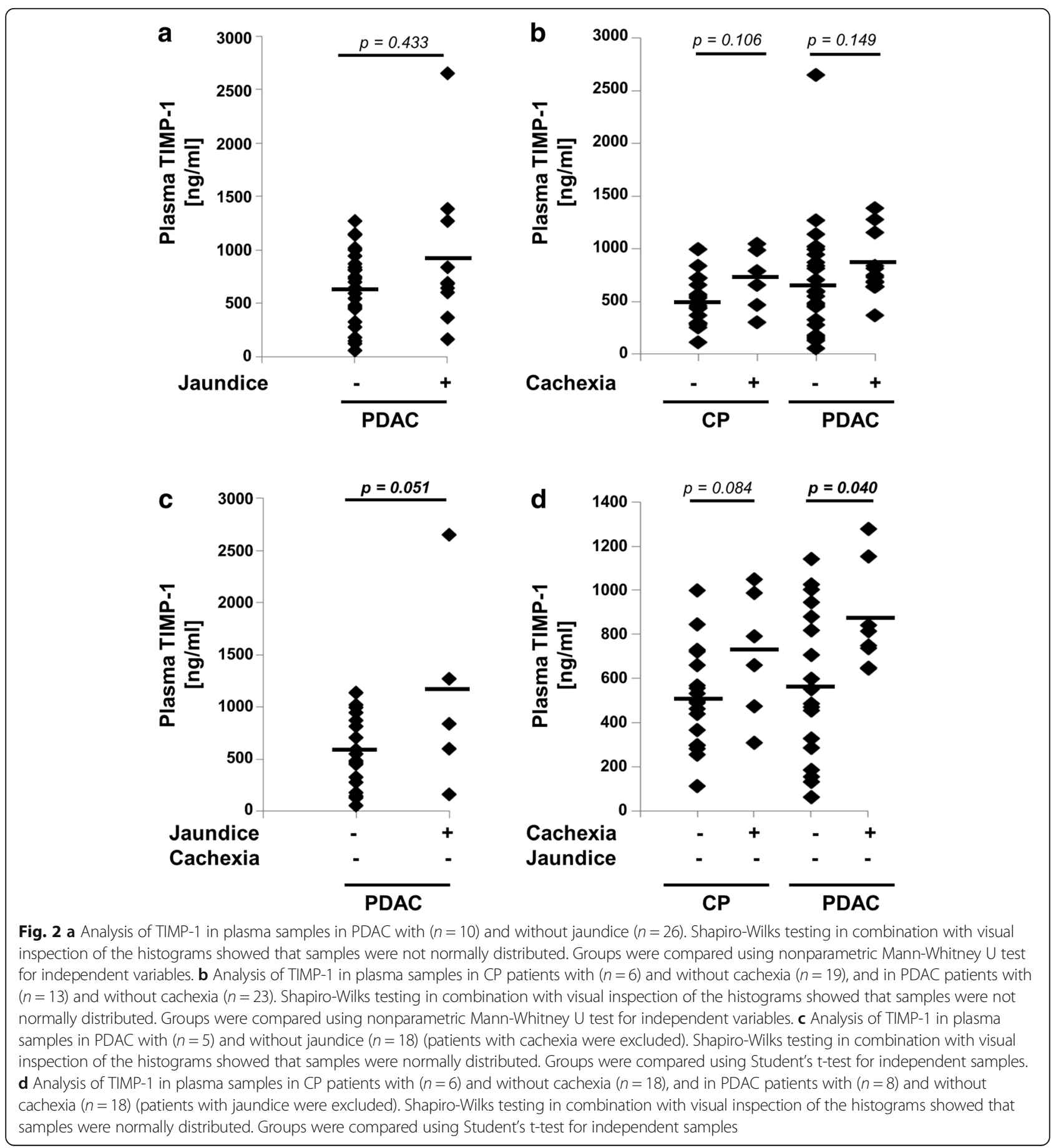


Table 3 Correlations between plasma TIMP-1 and selected parameters of cachexia

\begin{tabular}{|c|c|c|c|c|c|c|c|c|c|c|c|c|}
\hline & & ASA & Height, m & Weight, kg & $\mathrm{BMI}, \mathrm{kg} / \mathrm{m} 2$ & $\begin{array}{l}\text { Weight } \\
\text { loss, kg }\end{array}$ & $\begin{array}{l}\text { Weight } \\
\text { loss, \% }\end{array}$ & $\begin{array}{l}\text { Cachexia } \\
\text { (yes or no) }\end{array}$ & $\begin{array}{l}\text { Lung } \\
\text { function: } \\
\text { FEV1, I }\end{array}$ & $\begin{array}{l}\text { Lung } \\
\text { function: } \\
\text { FEV1, \% }\end{array}$ & $\begin{array}{l}\text { Lung } \\
\text { function: } \\
\text { FVC, I }\end{array}$ & $\begin{array}{l}\text { Lung } \\
\text { function } \\
\text { FVC, \% }\end{array}$ \\
\hline \multirow[t]{3}{*}{$\begin{array}{l}\text { Plasma TIMP-1, } \\
\mathrm{ng} / \mathrm{ml}\end{array}$} & $\begin{array}{l}\text { Spearman's } \\
\text { correlation }\end{array}$ & .305 & -.217 & .028 & .161 & .304 & .281 & .293 & -.374 & -.246 & -.546 & -.305 \\
\hline & $\begin{array}{l}\text { Significance } \\
\text { (2-tailed) }\end{array}$ & .027 & .099 & .831 & .224 & .017 & .034 & .020 & .035 & .175 & .001 & .089 \\
\hline & $\mathrm{N}$ & 53 & 59 & 59 & 59 & 61 & 57 & 63 & 32 & 32 & 32 & 32 \\
\hline
\end{tabular}

was no significant difference in the distribution of patients according to American Society of Anesthesiologists (ASA) physical status stages (Table 1).

TIMP-1 was previously shown to be of prognostic and diagnostic value in PDAC $[15,16])$. First, we tested this observation in our patient cohort and confirmed that plasma TIMP-1 levels were significantly higher in PDAC patients compared to healthy controls and CP. This was true when comparing all PDAC $(p=0.021 ; p=0.056)$ as well as PDAC patients with UICC stage $4(p=0.01$; $p=0.009$ ) (Fig. 1a). TIMP-1 significantly correlates with the presence of liver metastases (Table 2). The cut-off value of plasma TIMP-1 concentrations was defined as $842 \mathrm{ng} / \mathrm{ml}$ using maximally selected log-rank statistics performed by $\mathrm{R}$ Software. In PDAC patients, nine out of 36 (25\%) had higher plasma TIMP-1 levels than the cut off value. The median overall survival (OS) was significantly higher in patients with low plasma TIMP-1 levels ( $n=26,453$ days [440-768] 95\% CI] as compared to patients with high plasma TIMP-1 levels $(n=9,202$ days $[117-817,95 \% \mathrm{CI}](p<0.001)$. The Kaplan Meier analysis did not reach statistical significance in our cohort ( $p=0.093$, Kaplan Meier, log-rank test) (Fig. 1b).

\section{TIMP-1 levels in patients with jaundice and cachexia}

PDAC-related conditions can influence TIMP-1 levels secondary to PDAC and could therefore interfere with the use of TIMP-1 as a biomarker. Consistent with previous reports, [22] we confirmed mostly higher plasma TIMP-1 levels in PDAC patients with jaundice $(n=10)$ compared to patients without jaundice $(n=26)$ in our cohort (Fig. 2a). We also observed increased TIMP-1
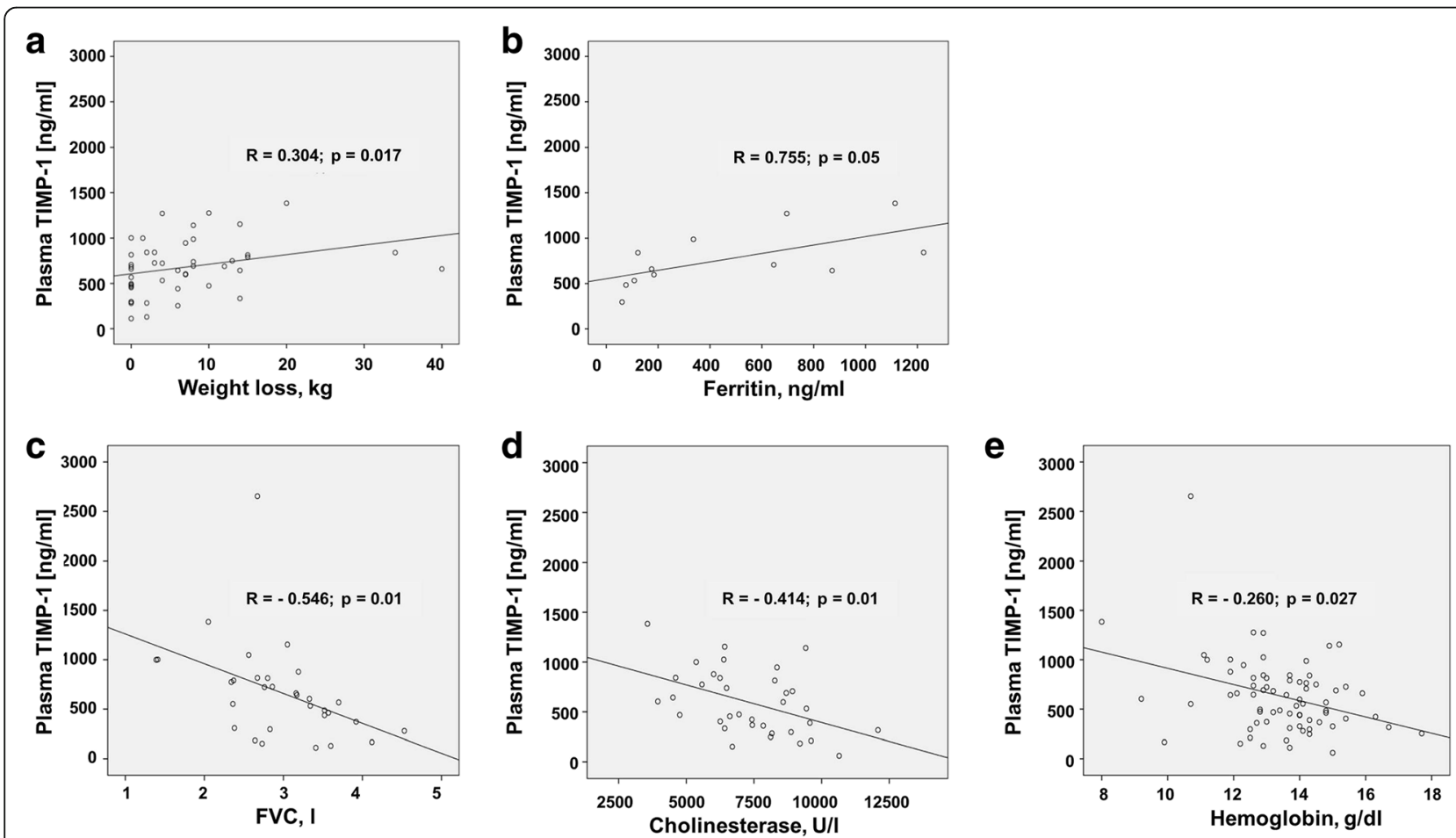

Fig. 3 Associations between plasma TIMP-1 and weight loss (a), ferritin (b), FVC (c) cholinesterase (d) and hemoglobin (e) were tested by Spearman correlations 


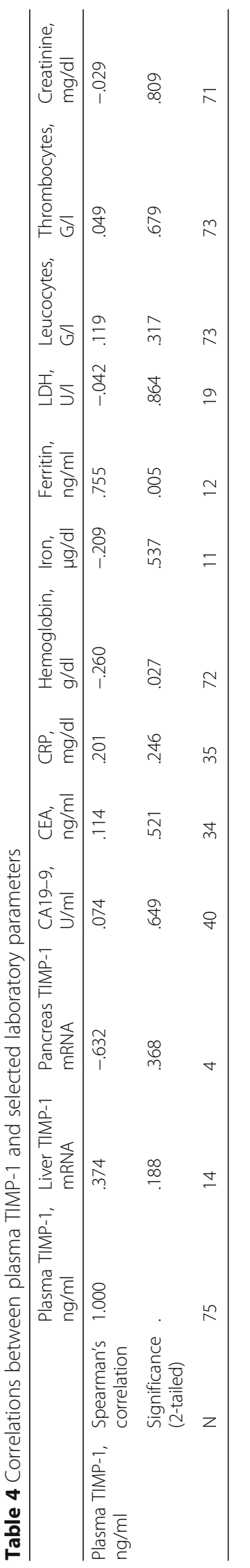


plasma levels in $\mathrm{CP}$ and in PDAC patients with cachexia compared to those without, although these differences were not statistically significant $(p=0.149)$ (Fig. 2b). We then separated the effects of jaundice and cachexia within our cohort to test their individual associations with TIMP-1 levels. In non-cachectic patients, TIMP-1 plasma levels were significantly associated with jaundice (Fig. 2c). Furthermore, exclusion of jaundice patients revealed a clear association of TIMP-1 plasma levels with cachexia in CP and PDAC patients (Fig. 2d). Thus, plasma TIMP-1 levels are elevated in both, cachexia and jaundice patients due to the individual condition.

\section{TIMP-1 and clinical parameters of cancer cachexia}

Next, we tested whether elevated TIMP-1 levels in cachexia may suggest a potential use as cachexia biomarker. We assessed clinical markers of cachexia and presence of cachexia in our patient cohort. There was a significant difference between the absolute and relative weight loss within the six months before diagnosis when comparing the cachexia and the non-cachexia PDAC group $(p<0.001)$. The mean value ( \pm standard deviation) for the absolute weight loss was $2.3 \pm 2.7 \mathrm{~kg}$ for non-cachexia patients and $13.7 \pm 7.4 \mathrm{~kg}$ for cachexia patients. The mean value for the relative weight loss was $3.0 \pm 3.5 \%$ for noncachexia patients and $16.0 \pm 6.4 \%$ for cachexia patients. The mean OS in patients with cachexia was lower, albeit not statistically significant ( $480 \pm 68$ versus $621 \pm 104$ days, $p=0.801)$. The M. psoas cross-sectional area was significantly lower in PDAC patients with cachexia as compared to controls and PDAC patients without cachexia ( $p=0.035$ and $p=0.031$, respectively) (Table 1$)$. The thickness of $M$. erector spinae and $M$. psoas was reduced in $\mathrm{CP}$ patients with cachexia as compared to $\mathrm{CP}$ patients without cachexia (Table 1). Elevated plasma TIMP-1 levels were significantly correlated with absolute $(p=0.017)$ (Table 3 , Fig. 3a) and relative $(p=0.034)$ weight loss, and with ferritin (Table 4, Fig. 3b) and inversely correlated with parameters of lung function, FEV1 $(p=0.035)$ (Table 3, Fig. 3c) and FVC $(p=0.01)$, cholinesterase levels (Table 5, Fig. 3d) and hemoglobin levels (Table 4, Fig. 3e). This shows a clear association of TIMP-1 plasma levels with clinical markers of cachexia.

\section{Influence of cachexia and jaundice on TIMP-1 as biomarker} in PDAC

In the final set of analyses, we tested whether alterations in TIMP-1 plasma levels in patients with cachexia and jaundice may limit its use as a biomarker in PDAC. When patients with cachexia (Fig. 4a) or patients with jaundice (Fig. 4b) were excluded, we still observed a stepwise increase of TIMP-1 levels from healthy individuals to patients with $\mathrm{CP}$ and PDAC, but the differences became less drastic as compared to Fig. 1a. Interestingly, both low TIMP-1 levels and absence of cachexia were independently beneficial for survival (Fig. 4c, curve \#1 vs. curve \#4). Combining the two parameters (low TIMP-1 levels and absence of cachexia) in the survival analysis yielded an improved prognostic value (Fig. $4 \mathrm{~d}$ curve \#1) as compared to use of TIMP-1 levels alone (Fig. 1b). In contrast, jaundice interfered with the usefulness of TIMP-1 as a prognostic marker; systemic TIMP-1 levels showed a clear association with survival only when patients with jaundice were excluded (Fig. 4e curve \#1 vs. curve \#3). Combining both parameters diminished association of TIMP-1 levels with survival time (Fig. 4f). Thus, TIMP-1 only predicted survival in the absence of jaundice in our cohort while accounting for cachexia improved its prognostic value.

\section{Discussion}

Our study focused on plasma TIMP-1 levels as a clinical biomarker in patients with $\mathrm{CP}$ and PDAC in the context of cachexia and jaundice. We report that TIMP-1 was associated with clinical markers of cachexia and also with the presence of cachexia but only in patients without jaundice. Furthermore, TIMP-1 appears to be counterindicated as a survival marker in patients with jaundice, while TIMP-1 and cachexia may be a promising combination of prognostic markers.

We are the first to report a possible association of TIMP-1 and cachexia in patients. Several laboratory (hemoglobin, ferritin, serum cholinesterase) and clinical parameters (relative and absolute weight loss, spirometry tests FEV1 and FVC) known to be associated with cachexia exhibited a significant correlation with plasma TIMP-1 levels in our study. We also found significantly higher TIMP-1 levels in patients with cachexia in the

Table 5 Correlations between plasma TIMP-1 and parameters of liver function

\begin{tabular}{|c|c|c|c|c|c|c|c|c|c|c|}
\hline & & $\begin{array}{l}\text { Bilirubin, } \\
\mathrm{mg} / \mathrm{dl}\end{array}$ & $\begin{array}{l}\text { Albumin, } \\
\text { g/dl }\end{array}$ & $\begin{array}{l}\text { Protein, } \\
\text { g/dl }\end{array}$ & $\begin{array}{l}\text { Alkaline } \\
\text { phosphatase, U/I }\end{array}$ & $\begin{array}{l}\text { Cholinesterase, } \\
\text { U/l }\end{array}$ & Quick, \% & GOT, U/I & GPT, U/I & GGT, U/I \\
\hline \multirow[t]{3}{*}{$\begin{array}{l}\text { Plasma TIMP-1, } \\
\mathrm{ng} / \mathrm{ml}\end{array}$} & $\begin{array}{l}\text { Spearman's } \\
\text { correlation }\end{array}$ & .033 & -.112 & -.270 & .228 & -.414 & -.098 & .121 & .166 & .184 \\
\hline & $\begin{array}{l}\text { Significance } \\
\text { (2-tailed) }\end{array}$ & .785 & .543 & .096 & .101 & .010 & .419 & .434 & .172 & .124 \\
\hline & $N$ & 72 & 32 & 39 & 53 & 38 & 70 & 44 & 69 & 71 \\
\hline
\end{tabular}



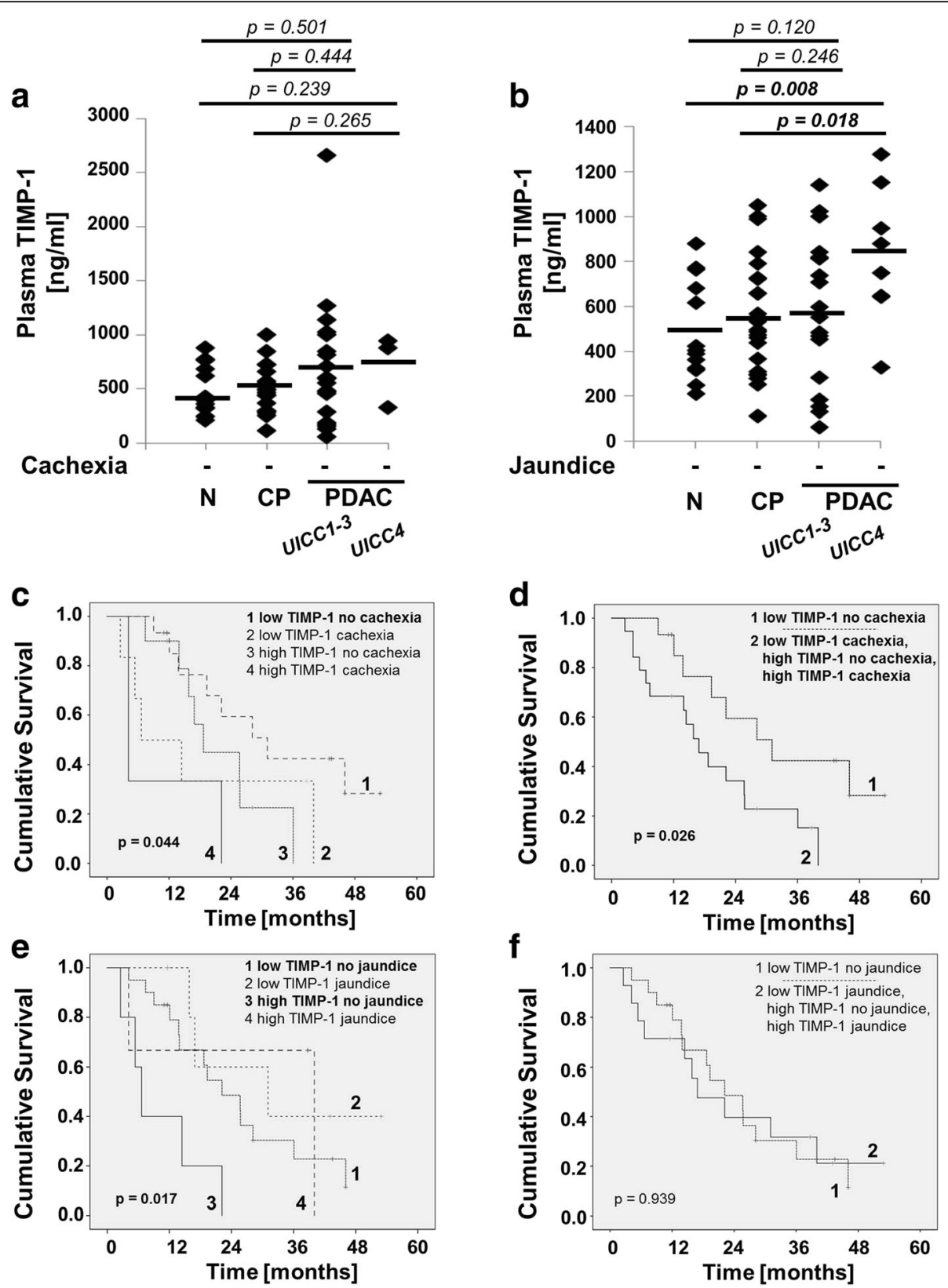

Fig. 4 a Analysis of TIMP-1 in healthy individuals (control, $n=13)$, in CP $(n=19)$, PDAC patients UICC $1-3(n=20)$, and PDAC patients UICC 4 $(n=3)$ (patients with cachexia were excluded). Shapiro-Wilks testing in combination with visual inspection of the histograms showed that samples were not normally distributed. Groups were compared using nonparametric Mann-Whitney U test for independent variables. b Analysis of TIMP-1 in patients without tumor and acute inflammatory disease (control, $n=13)$, in CP $(n=24)$, PDAC patients UICC 1-3 ( $n=18)$, and PDAC patients UICC $4(n=8)$ (patients with jaundice were excluded). Shapiro-Wilks testing in combination with visual inspection of the histograms showed that samples were normally distributed. Groups were compared using Student's t-test for independent samples. c-f Relation of plasma TIMP-1 and PDAC-associated cachexia $(\mathbf{c}, \mathbf{d})$ and jaundice $(\mathbf{e}, \mathbf{f})$ to Kaplan Meier Overall Survival (OS) curves in PDAC patients. For the optimal cut-off at $842 \mathrm{ng} / \mathrm{ml}$ (see Fig. 1b) and presence or absence of cachexia and jaundice the Kaplan Meier curves were generated. Differences were analyzed by the Log-rank (Mantel-cox) test. $\mathbf{c} p=0.112$ low TIMP-1 no cachexia vs. low TIMP-1 cachexia; $p=0.097$ low TIMP-1 no cachexia vs. high TIMP-1 no cachexia; $\boldsymbol{p}=\mathbf{0 . 0 1 4}$ low TIMP-1 no cachexia vs. high TIMP-1 cachexia; $p=0.978$ low TIMP-1 cachexia vs. high TIMP-1 no cachexia; $p=0.095$ low TIMP-1 cachexia vs. high TIMP-1 cachexia; $p=0.402$ high TIMP-1 no cachexia vs. high TIMP-1 cachexia. e $p=0.322$ low TIMP-1 no jaundice vs. low TIMP-1 jaundice; $\boldsymbol{p}=\mathbf{0 . 0 1 0}$ low TIMP-1 no jaundice vs. high TIMP-1 no jaundice; $p=0.670$ low TIMP-1 no jaundice vs. high TIMP-1 jaundice; $\boldsymbol{p}=\mathbf{0 . 0 1 4}$ low TIMP-1 jaundice vs. high TIMP-1 no jaundice; $p=0.766$ low TIMP-1 jaundice vs. high TIMP-1 jaundice; $p=0.152$ high TIMP-1 no jaundice vs. high TIMP-1 jaundice 
jaundice-free subgroup of our cohort. As plasma TIMP1 levels were elevated in both, cachexia and jaundice patients due to the individual conditions, it is likely that the effects of concomitant jaundice weaken the association between TIMP-1 and cachexia. There is an urgent need to develop suitable biomarkers and potential drug targets for cancer cachexia which will facilitate better definition and earlier diagnosis of the syndrome. It is tempting to propose the use of TIMP-1 as cachexia marker, i.e. due to its involvement in muscle remodeling in cancer cachexia [26], which may allow for detection of early muscle wasting in on-setting cachexia. Future studies would benefit from increasing our patient cohort and incorporating continuous scoring methods for cachexia staging, such as CASCO score [37].

We show for the first time that TIMP-1 may be a useful prognostic marker especially in combination with cachexia but notably, not in patients with jaundice. There is a large body of evidence showing that systemic TIMP-1 levels have prognostic value in pancreatic cancer $[15,16,21,38]$. PDAC-related conditions such as jaundice and cachexia are associated with tissue damage, which can influence TIMP-1 levels secondary to PDAC and could thus interfere with the use of TIMP-1 as a biomarker in PDAC. It was indeed reported that patients with PDAC-induced jaundice have significantly elevated levels of TIMP-1, compared to PDAC patients without jaundice, and that even benign jaundice can lead to increased TIMP-1 levels [22]. Although TIMP-1 levels were significantly raised at the time of diagnosis in cancer patients compared to healthy controls, the absolute increase observed in the absence of biliary obstruction was relatively small compared to those seen in the presence of obstruction [22]. Our results confirm this finding, demonstrating that differences in plasma TIMP-1 between PDAC, CP, and control patients are less drastic when patients with jaundice or cachexia are excluded. TIMP-1 could thus be overestimated as a diagnostic marker in case PDAC-related jaundice and cachexia are not accounted for. This is by the observation that jaundice and cachexia patients had higher plasma TIMP-1 level. Interestingly, this increase in TIMP-1 levels was similar in both conditions but had clearly different consequences for the use of TIMP-1 as prognostic factor: Presence of jaundice clearly interfered with the prognostic value of TIMP-1, and the impact of TIMP-1 on survival became most evident when jaundice patients were excluded. We thus emphasize that it is essential to consider whether or not a patient has jaundice when using TIMP-1 as a biomarker. In fact, this limitation is not restricted to TIMP-1 and CA19-9, the most established tumor marker for PDAC with a reported sensitivity of $79 \%$ and specificity of $82 \%$ [39], was shown to be influenced by jaundice [40]. There is also a positive correlation between bilirubin and CA19-9 in benign jaundice whereas no such relationship exists for malignant jaundice cases [40]. In contrast, the higher TIMP-1 levels of cachexia patients did not interfere with usefulness of TIMP-1 as prognostic marker and excluding cachectic patients even reduced the association of TIMP-1 with survival. Moreover, combining TIMP-1 plasma levels with cachexia status improved the prognostic value, suggesting this newly identified association between TIMP-1 and cachexia could provide a benefit for patient stratification.

\section{Conclusions}

We show that TIMP-1 was counterindicated as a marker in patients with jaundice, while TIMP-1 together with cachexia appeared as a promising combination of prognostic parameters. We report for the first time that TIMP-1 was associated with presence of cachexia and cachexiaassociated clinical markers, and conclude that TIMP-1 should be further evaluated as a cachexia biomarker. We emphasize that careful clinical evaluation of the patient, under consideration of PDAC-related secondary conditions, must be recognized as the basis for a meaningful interpretation of molecular biomarkers.

\section{Abbreviations \\ BMI: Body mass index; CRP: c-reactive protein; CT: Computed tomography; $\mathrm{FEV}_{1}$ : Forced expiratory volume at the end of the first second of forced expiration; FVC: Forced vital capacity; PDAC: Pancreatic ductal adenocarcinoma; TIMP-1: Tissue inhibitor of metalloproteinases-1; UICC: the International Union against Cancer}

\section{Acknowledgements}

We thank Dr. Markus Thaler from the Institute of Clinical Chemistry and Pathobiochemistry, Klinikum rechts der Isar, for assistance in the section on laboratory examinations. We thank Dr. Alison Casey and Dr. Khalid O. Alfarouk for thorough proofreading of the manuscript.

\section{Funding}

This work was supported by grants to AK from the Deutsche Forschungsgemeinschaft (KR2047/1-2, KR2047/1-3, and KR2047/3-1), and the European Union Seventh Framework Programme (FP7/2007-2013 n²63307). This work was partly supported by grant 2016.124.1 of the Wilhelm-SanderStiftung to A.K.

\section{Availability of data and materials}

The datasets generated during and/or analysed during the current study are available from the corresponding author on reasonable request.

\section{Authors' contributions}

$\mathrm{OP}, \mathrm{BG}$, and $\mathrm{AK}$ designed the study and wrote the paper. BG performed the ELISA experiments. OP and OLP participated in ELISA experiments. UN performed survival and cut-off analysis and edited the manuscript. OP and OLP were responsible for the clinical data acquisition. CJ provided survival data and edited the manuscript. ECS measured fat and muscle tissue on computed tomography scans. HF and MEM helped providing the study tissues and editing the manuscript. All authors read and approved the final version of manuscript.

\section{Ethics approval and consent to participate}

The clinical and laboratory data could be used only after the consent of the patient to other scientific purposes. An Institutional Review Board „Ethikkommission der Technischen Universität München "approved the study (Nr. 1946/07). Written informed consent was obtained from the human subjects. 


\section{Consent for publication}

Not applicable.

\section{Competing interests}

The authors have declared no conflicts of interest.

\section{Publisher's Note}

Springer Nature remains neutral with regard to jurisdictional claims in published maps and institutional affiliations.

\section{Author details}

${ }^{1}$ Klinik und Poliklinik für Chirurgie, Klinikum rechts der Isar, Technische Universität München, Ismaninger Str. 22, 81675 Munich, Germany. ${ }^{2}$ Institut für Molekulare Immunologie und Experimentelle Onkologie, Technische Universität München, Munich, Germany. ${ }^{3}$ Medizinische Klinik II, Klinikum Obergöltzsch, Rodewisch, Germany. ${ }^{4}$ Institut für Radiology, Klinikum rechts der Isar, Technische Universität München, Munich, Germany.

\section{Received: 28 November 2016 Accepted: 25 January 2018}

\section{Published online: 02 February 2018}

\section{References}

1. Seubert B, Grunwald B, Kobuch J, Cui H, Schelter F, Schaten S, et al. Tissue inhibitor of metalloproteinases (TIMP)-1 creates a premetastatic niche in the liver through SDF-1/CXCR4-dependent neutrophil recruitment in mice. Hepatology. 2015;61(1):238-48.

2. Holten-Andersen MN, Stephens RW, Nielsen HJ, Murphy G, Christensen IJ, Stetler-Stevenson W, et al. High preoperative plasma tissue inhibitor of metalloproteinase-1 levels are associated with short survival of patients with colorectal cancer. Clin Cancer Res. 2000:6:4292-9.

3. Wurtz SO, Moller S, Mouridsen H, Hertel PB, Friis E, Brunner N. Plasma and serum levels of tissue inhibitor of metalloproteinases-1 are associated with prognosis in node-negative breast cancer: a prospective study. Mol Cell Proteomics. 2008:7:424-30.

4. Kozlowski M, Laudanski W, Mroczko B, Szmitkowski M, Milewski R, Lapuc G. Serum tissue inhibitor of metalloproteinase 1 (TIMP-1) and vascular endothelial growth factor a (VEGF-A) are associated with prognosis in esophageal cancer patients. Adv Med Sci. 2013;58:227-34.

5. Cheng G, Fan X, Hao M, Wang J, Zhou X, Sun X. Higher levels of TIMP-1 expression are associated with a poor prognosis in triple-negative breast cancer. Mol Cancer. 2016;15:30

6. Song $G$, Xu S, Zhang H, Wang Y, Xiao C, Jiang T, et al. TIMP1 is a prognostic marker for the progression and metastasis of colon cancer through FAKPI3KAKT and MAPK pathway. J Exp Clin Cancer Res. 2016;35:148.

7. Zeng ZS, Cohen AM, Zhang ZF, Stetler-Stevenson W, Guillem JG. Elevated tissue inhibitor of metalloproteinase 1 RNA in colorectal cancer stroma correlates with lymph node and distant metastases. Clin Cancer Res. 1995;1:899-906.

8. Alpizar-Alpizar W, Laerum OD, Christensen IJ, Ovrebo K, Skarstein A, HoyerHansen $\mathrm{G}$, et al. Tissue inhibitor of Metalloproteinase-1 is confined to tumor-associated Myofibroblasts and is increased with progression in gastric adenocarcinoma. J Histochem Cytochem. 2016;64:483-94.

9. Yoshikawa T, Cho H, Tsuburaya A, Kobayashi O. Impact of plasma tissue inhibitor of metalloproteinase-1 on long-term survival in patients with gastric cancer. Gastric Cancer. 2009;12:31-6.

10. An HJ, Lee YJ, Hong SA, Kim JO, Lee KY, Kim YK, et al. The prognostic role of tissue and serum MMP-1 and TIMP-1 expression in patients with nonsmall cell lung cancer. Pathol Res Pract. 2016;212:357-64.

11. Cui H, Seubert B, Stahl E, Dietz H, Reuning U, Moreno-Leon L, et al. Tissue inhibitor of metalloproteinases-1 induces a pro-tumourigenic increase of miR-210 in lung adenocarcinoma cells and their exosomes. Oncogene. 2015;34:3640-50

12. Cui H, Grosso S, Schelter F, Mari B, Kruger A. On the pro-metastatic stress response to cancer therapies: evidence for a positive co-operation between TIMP-1, HIF-1alpha, and miR-210. Front Pharmacol. 2012;3:134.

13. Kruger A. Premetastatic niche formation in the liver: emerging mechanisms and mouse models. J Mol Med (Berl). 2015;93:1193-201.

14. Grunwald B, Harant V, Schaten S, Fruhschutz M, Spallek R, Hochst B, et al. Pancreatic pre-malignant lesions secrete TIMP1, which activates hepatic stellate cells via CD63 signaling to create a pre-metastatic niche in the liver. Gastroenterology. 2016;151(5):1011-24
15. Mroczko B, Lukaszewicz-Zajac M, Wereszczynska-Siemiatkowska U, Groblewska M, Gryko M, Kedra B, et al. Clinical significance of the measurements of serum matrix metalloproteinase-9 and its inhibitor (tissue inhibitor of metalloproteinase-1) in patients with pancreatic cancer: metalloproteinase-9 as an independent prognostic factor. Pancreas. 2009;38:613-8.

16. Poruk KE, Firpo MA, Scaife CL, Adler DG, Emerson LL, Boucher KM, et al. Serum osteopontin and tissue inhibitor of metalloproteinase 1 as diagnostic and prognostic biomarkers for pancreatic adenocarcinoma. Pancreas. 2013;42:193-7.

17. Gress TM, Muller-Pillasch F, Lerch MM, Friess H, Buchler M, Adler G. Expression and in-situ localization of genes coding for extracellular matrix proteins and extracellular matrix degrading proteases in pancreatic cancer. Int J Cancer. 1995;62:407-13.

18. Gong YL, Xu GM, Huang WD, Chen LB. Expression of matrix metalloproteinases and the tissue inhibitors of metalloproteinases and their local invasiveness and metastasis in Chinese human pancreatic cancer. J Surg Oncol. 2000;73:95-9.

19. Zhang $H$, Liu B, Xu XF, Jiang TT, Zhang XQ, Shi YL, et al. Pathophysiology of chronic pancreatitis induced by dibutyltin dichloride joint ethanol in mice. World J Gastroenterol. 2016:22:2960-70.

20. Pan S, Chen R, Crispin DA, May D, Stevens T, Mclntosh MW, et al. Protein alterations associated with pancreatic cancer and chronic pancreatitis found in human plasma using global quantitative proteomics profiling. Proteome Res. 2011;10:2359-76.

21. Pan S, Chen R, Brand RE, Hawley S, Tamura Y, Gafken PR, et al. Multiplex targeted proteomic assay for biomarker detection in plasma: a pancreatic cancer biomarker case study. J Proteome Res. 2012;11:1937-48.

22. Jenkinson C, Elliott V, Menon U, Apostolidou S, Fourkala OE, Gentry-Maharaj A, et al. Evaluation in pre-diagnosis samples discounts ICAM-1 and TIMP-1 as biomarkers for earlier diagnosis of pancreatic cancer. J Proteome. 2015;113:400-2.

23. Bachmann J, Buchler MW, Friess H, Martignoni ME. Cachexia in patients with chronic pancreatitis and pancreatic cancer: impact on survival and outcome. Nutr Cancer. 2013:65:827-33.

24. Springer J, Tschirner A, Haghikia A, von Haehling S, Lal H, Grzesiak A, et al. Prevention of liver cancer cachexia-induced cardiac wasting and heart failure. Eur Heart J. 2014;35:932-41.

25. Toneto AT, Ferreira Ramos LA, Salomao EM, Tomasin R, Aereas MA, GomesMarcondes MC. Nutritional leucine supplementation attenuates cardiac failure in tumour-bearing cachectic animals. J Cachexia Sarcopenia Muscle. 2016;7(5): $577-86$

26. Devine RD, Bicer S, Reiser PJ, Velten M, Wold LE. Metalloproteinase expression is altered in cardiac and skeletal muscle in cancer cachexia. Am J Physiol Heart Circ Physiol. 2015;309:H685-91.

27. Fearon $\mathrm{K}$, Strasser F, Anker SD, Bosaeus I, Bruera E, Fainsinger RL, et al. Definition and classification of cancer cachexia: an international consensus. Lancet Oncol. 2011:12:489-95.

28. Martignoni ME, Dimitriu C, Bachmann J, Krakowski-Rosen H, Ketterer K, Kinscherf $\mathrm{R}$, et al. Liver macrophages contribute to pancreatic cancer-related cachexia. Oncol Rep. 2009;21:363-9.

29. Skorokhod A, Bachmann J, Giese N, Martignoni ME, Krakowski-Roosen H. Real-imaging CDNA-AFLP transcript profiling of pancreatic cancer patients: Egr1 as a potential key regulator of muscle cachexia. BMC Cancer. 2012;12:265.

30. Schwarz S, Prokopchuk O, Esefeld K, Groschel S, Bachmann J, Lorenzen S, et al. The clinical picture of cachexia: a mosaic of different parameters (experience of 503 patients). BMC Cancer. 2017;17:130.

31. Bachmann J, Heiligensetzer M, Krakowski-Roosen $H$, Buchler MW, Friess $H$, Martignoni ME. Cachexia worsens prognosis in patients with resectable pancreatic cancer. J Gastrointest Surg. 2008;12:1193-201.

32. Bachmann J, Ketterer K, Marsch C, Fechtner K, Krakowski-Roosen H, Buchler MW, et al. Pancreatic cancer related cachexia: influence on metabolism and correlation to weight loss and pulmonary function. BMC Cancer. 2009;9:255

33. Prokopchuk O, Steinacker J, Nitsche U, Otto S, Bachmann J, Schubert E, et al. IL-4 mRNA is downregulated in the liver of pancreatic cancer patients suffering from cachexia. Nutr Cancer. 2016:69 in press

34. Hothorn T, Zeileis A. Generalized maximally selected statistics. Biometrics. 2008:64:1263-9.

35. Cascinu S, Falconi M, Valentini V, Jelic S, Group EGW. Pancreatic Cancer: ESMO clinical practice guidelines for diagnosis, treatment and follow-up. Ann Oncol. 2010;21(Suppl 5):v55-8.

36. Hartwig W, Buchler MW. Pancreatic cancer: current options for diagnosis, staging and therapeutic management. Gastrointest Tumors. 2013;1:41-52. 
37. Argiles JM, Lopez-Soriano FJ, Toledo M, Betancourt A, Serpe R, Busquets S. The cachexia score (CASCO): a new tool for staging cachectic cancer patients. J Cachexia Sarcopenia Muscle. 2011;2:87-93.

38. Joergensen MT, Brunner N, De Muckadell OB. Comparison of circulating MMP-9, TIMP-1 and CA19-9 in the detection of pancreatic cancer. Anticancer Res. 2010;30:587-92.

39. Goonetilleke KS, Siriwardena AK. Systematic review of carbohydrate antigen (CA 19-9) as a biochemical marker in the diagnosis of pancreatic cancer. Eur J Surg Oncol. 2007;33:266-70.

40. Mann DV, Edwards R, Ho S, Lau WY, Glazer G. Elevated tumour marker CA19-9: clinical interpretation and influence of obstructive jaundice. Eur J Surg Oncol. 2000;26:474-9.

Submit your next manuscript to BioMed Central and we will help you at every step:

- We accept pre-submission inquiries

- Our selector tool helps you to find the most relevant journal

- We provide round the clock customer support

- Convenient online submission

- Thorough peer review

- Inclusion in PubMed and all major indexing services

- Maximum visibility for your research

Submit your manuscript at www.biomedcentral.com/submit
Biomed Central 